INPLASY

PROTOCOL

To cite: Li et al. Lung

ultrasound guided therapy for

heart failure: an updated meta-

analyses and trial sequential

analysis. Inplasy protocol

202220124. doi:

10.37766/inplasy2022.2.0124

Received: 28 February 2022

Published: 28 February 2022

Corresponding author:

Peng Li

lipbenzi@126.com

Author Affiliation:

Beijing Hospital, National

Center of Gerontology,

Institute of Geriatric

Medicine, Chinese Academy

of Medical ScienceNational.

Support: Beijing Hospital

Clinical Research.

Review Stage at time of this submission: Data extraction.

Conflicts of interest:

None declared.

\section{Lung ultrasound guided therapy for heart failure: an updated meta-analyses and trial sequential analysis}

Li, P1; Ren, J2; Li, Y33.

Review question / Objective: We aim to evaluate the effect of lung ultrasound (LU) guided therapy on the rates of adverse cardiac events (MACE) in heart failure (HF) patients.

Condition being studied: Previous studies have found that Blines assessed by lung ultrasound can be used for risk stratification in patients with HF and to predict the occurrence of adverse cardiac events. Therefore, similar to BNP, lung ultrasound has clinical value in guiding the management of patients with HF. However, the role of LU in guiding HF therapy is still controversial. Moreover, previous study's samples are too small to explain the over clinical outcomes. Besides, previous meta-analyses study did not perform metaregression and/or subgroup analyses, or further analyze other parameters, such as heart function, quality of life and length of hospital stay.

INPLASY registration number: This protocol was registered with the International Platform of Registered Systematic Review and Meta-Analysis Protocols (INPLASY) on 28 February 2022 and was last updated on 28 February 2022 (registration number INPLASY202220124).

\section{INTRODUCTION}

Review question / Objective: We aim to evaluate the effect of lung ultrasound (LU) guided therapy on the rates of adverse cardiac events (MACE) in heart failure (HF) patients.
Rationale: In patients with acute HF (AHF), B-lines $\geq 15$ on 28 -zone lung ultrasound (LU) at discharge marked those with an increased five-fold risk for HF readmission or mortality. Meanwhile, in ambulatory patients with chronic HF (CHF), B-lines $\geq 3$ on five- or eight-zone LU identified patients with an nearly four-fold risk for 6-month HF 
hospitalization or mortality. Therefore, similar to BNP, lung ultrasound has clinical value in guiding the management of patients with HF. Therefore, As the evidence gathered has recently increased, we have performed a meta-analysis and trial sequential analysis (TSA) to evaluate the effect of LU-guided treatment on major adverse cardiac events (MACE) in patients with HF.

Condition being studied: Previous studies have found that B-lines assessed by lung ultrasound can be used for risk stratification in patients with HF and to predict the occurrence of adverse cardiac events. Therefore, similar to BNP, lung ultrasound has clinical value in guiding the management of patients with HF. However, the role of $L U$ in guiding HF therapy is still controversial. Moreover, previous study's samples are too small to explain the over clinical outcomes. Besides, previous metaanalyses study did not perform metaregression and/or subgroup analyses, or further analyze other parameters, such as heart function, quality of life and length of hospital stay.

\section{METHODS}

Search strategy: We searched MEDLINE (source, PubMed from 2005 to December 2021), EMBASE (2005 to December 2021), the Cochrane Controlled Clinical Trials Register Database (to December 2021), Google Scholar (to December 2021), SinoMed (to December 2021) and the ClinicalTrials.gov website (to December 2021) using the terms "heart failure", "lung ultrasound", "heart failure visits", "heart failure rehospitalization", and "randomized trial". Manual reference checking of the bibliographies of all relevant articles was performed. No restrictions were applied. We will search articles in three electronic database including PubMed, EMBASE and Cochrane Library. All the English and Chinese publications until 31 December, 2021 will be searched without any restriction of countries or article type. Reference list of all selected articles will independently screened to identify additional studies left out in the initial search.

Participant or population: Patients with heart failure.

Intervention: Lung ultrasound and usual care-guided HF treatment.

Comparator: Usual care-guided HF treatment.

Study designs to be included: Randomized clinical trial.

Eligibility criteria: Trials were considered eligible if they met these criteria: 1) patients with AHF or CHF; 2) HF patients was treated guided by the LU and usual care versus usual care alone; 3) the primary outcome of interest was the rate of MACE, including HF related rehospitalization, symptomatic HF, or all-cause mortality; 4) the study was a RCT. Exclusion criteria were (1) patients with cardiac shock; (2) complicated with pneumonia; (3) singlearm study; (4) without primary outcome; (5) retrospective study, animal study, case report, or review; and (6) duplicated data.

Information sources: MEDLINE (source, PubMed), EMBASE, the Cochrane Controlled Clinical Trials Register Database, Google Scholar, SinoMed and the ClinicalTrials.gov website.

Main outcome(s): Primary outcome was the rate of MACE.

Additional outcome(s): Secondary outcomes were the rate of $\mathrm{HF}$ related rehospitalization, symptomatic HF, allcause mortality, the length of hospital stay, change of NT-proBNP, diuretic dosage, quality of life, and the rate of adverse events (acute kidney injury, hypokalemia).

Data management: Results were analyzed quantitatively with STATA 14.0 software (Stata Corp, Califonia, USA) using the fixedeffects model. Heterogeneity was examined by the 12 statistic and the chisquared test. A value of $12>50 \%$ was considered as a substantial level of 
heterogeneity. Once heterogeneity was noted, between-study sources of heterogeneity were investigated using subgroup analysis by stratifying original estimates according to study characteristics. Publication bias was assessed quantitatively using Egger's regression test $(P \leq 0.10)$ and qualitatively, by visual inspection of funnel plots of the logarithm of RRs versus their standard errors. Univariate meta-regression analysis was used to identify possible contributors to between-study variance. In particular, we investigated associations between the RR for MACE, HF related rehospitalization, symptomatic HF and clinically plausible factors, including acute heart failure, patients' number, age, AF, DM, ischemic HF, LVEF, TnI, eGFR, B lines, NT-proBNP and follow-up duration. Sensitivity analyses were conducted to determine the influence of individual trials on the overall pooled results. All analyses were performed according to the intention-to-treat principle. Statistical significance was set at 0.05 for the Z-test for RR.

Quality assessment / Risk of bias analysis: The Preferred Reporting Items for Systemic Reviews and Meta-Analyses (PRISMA) statement was followed. Two reviewers assessed the quality of the selected trials. Components used for quality assessment were means of generation of random sequence, allocation concealment, blinding of outcome assessment, and selective outcome reporting.

Strategy of data synthesis: We calculated the pooled relative risk (RR) for dichotomous outcomes and the standard mean difference (SMD), or weighted mean difference (WMD) for continuous data with 95\% confidence intervals (Cl).

Subgroup analysis: Based on the mean level of baseline clinical factors (age, AF, DM, ischemic HF, LVEF, Tnl, eGFR, B-lines, NT-proBNP, follow-up duration) and patients condition (acute heart failure, chronic heart failure), the patients' condition was divided into "acute heart failure" and "chronic heart failure"; age was classified into " $<70.0$ years" and " $\geq 70.0$ years"; AF was classified into " $<27.2 \%$ " and " $\geq 27.2 \%$ "; DM was classified into " $<38.3 \%$ " and " $\geq 38.3 \%$ "; ischemic HF was classified into " $<44.2 \%$ " and " $\geq 44.2 \%$ "; LVEF was classified into " $<37.5 \%$ " and " $\geq 37.5 \%$ "; Tnl was categorized as " $<1.23 \mathrm{ng} / \mathrm{ml}$ " and " $\geq 1.23 \mathrm{ng} / \mathrm{ml}$ "; eGFR was categorized as "<48.8 $\mathrm{ml} / \mathrm{min} / 1.73 \mathrm{~m} 2$ " and " $\geq 48.8 \mathrm{ml} / \mathrm{min} /$ $1.73 \mathrm{~m} 2$ "; B lines was reported as " $<5.0$ " and " $\geq 5.0$ "; follow-up duration was reported as " $<4.7$ months" and " $\geq 4.7$ months".

Sensitivity analysis: Sensitivity analyses were conducted to determine the influence of individual trials on the overall pooled results.

Language: English.

Country(ies) involved: China.

Keywords: Lung ultrasound; heart failure; adverse cardiac event; prognosis; metaanalysis.

Dissemination plans: This paper will be published in Theranostics.

Contributions of each author:

Author 1 - Peng Li - Author 1 drafted the manuscript.

Email: lipbenzi@126.com

Author 2 - Junhong Ren - The author provided statistical expertise.

Email: renjunhong2002@hotmail.com

Author 3 - Yan Li - The author contributed to the development of the selection criteria, and the risk of bias assessment strategy.

Email: since8895@126.com 